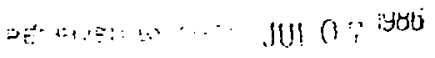

UCRL- -94116

DE86 012351

\title{
A HIGH INTENSITY LINE SOURCE FOR X-RAY SPECTROMETER CALIBRATION
}

\author{
R. S. Thoe
}

This paper was prepared for submittal to the Society for Photo-optical Instrumentation Engineers (SPIE) 30th Annual International Technical Symposium on Optical and Optoelectronic Applied Sciences and Engineering, San Diega, California, August 17-22, 1986

June 1986

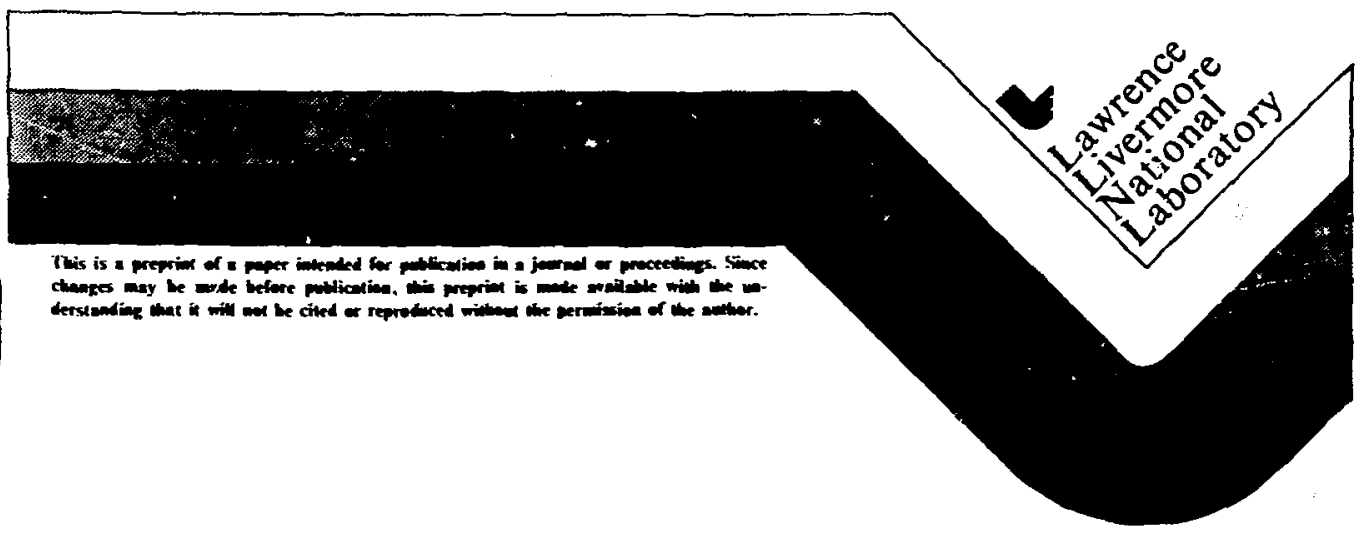




\section{R. S. Thoe}

Univeraity of California, Lawrence Livermece National Laboratory Mail Stop 43, P.O. Box 806, Livernore, California 94550

\section{NASTER}

\begin{abstract}
A high intensity electron-impact $x$-ray source using a one-dimensional Pierce lens has been built for the pupose of calibrating a bent crystal $x$-ray spectrometer. This source focuses up to $100 \mathrm{~mA}$ of $20-\mathrm{keV}$ electrons to a line on a liquid-cooled anode. The line (which can serve as a virtual slit for the specirometer) measures approximately $800 \mu \times 2 \mathrm{~cm}$.
\end{abstract}

The source is portable and therefore adaptable to numerous types of spectrometer applications. One particular application, the calibration of a high resolution $\left(r=10^{-1}\right)$ time-resolved crystal spectrometer, will be discussed in detail.

\title{
Introduction
}

High resolution $x$-ray spectra can reveal essential information about plasma density, radiation transport, and temperature. For many applications, however, the large distances at which the spectrometer must be located (i.e., $>10 \mathrm{~m}$ away from the plasma) can have a profound effect on its calibration and design. Such was the case in a recent experiment at the Nevada Test Site (NTS), in which I obtained time-resolved $x$-ray spectra in high resolution from a hot, dense, nuclear-driven plasma.

The experimental objectives were threefold: to obtain a spectral resolving power of 10,000 , an energy coverage of $20 \%$, and a time resolution of betler than $1 \mathrm{~ns}$. In meeting these objectives, the spectrometer design also had to meet the demands of an extremely hostile environment. Of course. most plasma diagnostics spectrometers are not exposed to the high radiation fields encountered in an NTS experiment. Nonetheless, the basic : ackground nijection and calibration techniques that I developed for this design can easily be applied to plasma spectroscopy in gereral.

\section{Spectrometer decign}

A basic design feature is the integration of the calibration facility inio the spectrometer itself. By this I mean that the $x$-ray source wis designed especially for the spectrometer, and the spectrometer was set up for in situ calibration using the $x$-ray source. Since specific details of this design will be published in a forthcoming joumal article, I will limit my remarks here to a brief summary of just the most important design characteristics. ${ }^{1}$

The source was to be located about $20 \mathrm{~m}$ away from the spectrometer. This large distance and the small angular extent of the source effectively ruliad out any johannson-type focusing geometry, while the need for a large spectral range ruled out the use of a flat crystal. ${ }^{2}$ I was committed from the start, therefore, to a bent crystal design.

In my forthcoming paper on spectrometer design, I show that bending a cystal decreases its sensitivity by an amount equal to (Rcos $\theta) / r$, where $r$ is the crystal-source distance, $R$ is the radius of curvature, and $\theta$ is the average Bragg angle. To make up for this decrease in sersitivity $f$ bent the crystal in the sagittal plane. In other words, the crystal was bent spherically rather than cylindrically, making the spectrometer similar in effect to a vent Von-Hamos.' At a Bragg angle of about 10 deg the astiguratism in this system gave ample separation between the sagittal and tangential focus so that high linear dispersion could be achieved.

Figure 1 is a drawing of the spectrometer and its detection system. Figure 2 shows the entire line of sight, from the plasma source to the imagescope, along with an enlarged sketch of the crystal optics (Detail A). Since any flexure of the diagnostics canister could affect energy calibration, a light source and linear reticon are used to monitur the alignment of the spectrometer during colibration and subsequent emplacement. The parameters of the quartz, image-scope, and fiber optic faceplate are given in Table 1.

Table 1. Spectrometer and Detection System Parameters

\begin{tabular}{|c|c|c|}
\hline Quartz $50 \overline{52}$ & Image-Scope & Fiber Optic Faceplate \\
\hline $\begin{array}{l}\mathrm{R}=88 \mathrm{~cm} \\
\mathrm{R}_{c}=6 \times 10^{-5} \\
\text { disp }=17 \mathrm{eV} / \mathrm{mm} \\
2 \mathrm{~d}=1.624 \mathrm{~A} \\
\theta=46.6 \mathrm{deg}\end{array}$ & $\begin{array}{l}\text { Length }=5 \mathrm{~m} \\
R=35 \mathrm{lp} / \mathrm{mm} \\
\text { transmission } \simeq 5 \% \\
\text { at } 380 \mathrm{~nm}\end{array}$ & $\begin{array}{l}\rho \simeq 23 \mathrm{mg} / \mathrm{cm}^{2} \\
\tau \simeq 0.7 \mathrm{~ns}\end{array}$ \\
\hline
\end{tabular}

The optical properties of this spectrometer are such that a poi.t source is brought to a line focus. At the tangenial focal phane this focus is achromatic, though it does suffer forn spherical aberration. The sagital focus on the other hand sulfers chromatic aberration (dispersion) but gives perfect stigmatic focusing in one dimension. In the forthcoming paper mentioned above, I approximate the dispersion as:

$$
\mathrm{dE} / \mathrm{d} \mathbf{z}=2 \cdot 2 \mathrm{~d}^{\prime} / \mathrm{R}
$$

where $\mathrm{dE} / \mathrm{dz}$ is the reciproal linear dispersion, and $2 \mathrm{~d}^{\prime}$ is the crystal $2 \mathrm{~d}$-space in eV (i.e., he/2d). The Bargg crystal for this spectrometer was quartz 5052 . With $2 d^{\prime}=7634 \mathrm{eV}$, and $R=88 \mathrm{~cm}$, this crystal gives a reciprocal dispersion of roughly $174 \mathrm{eV} / \mathrm{cm}$. Since the slit measured 


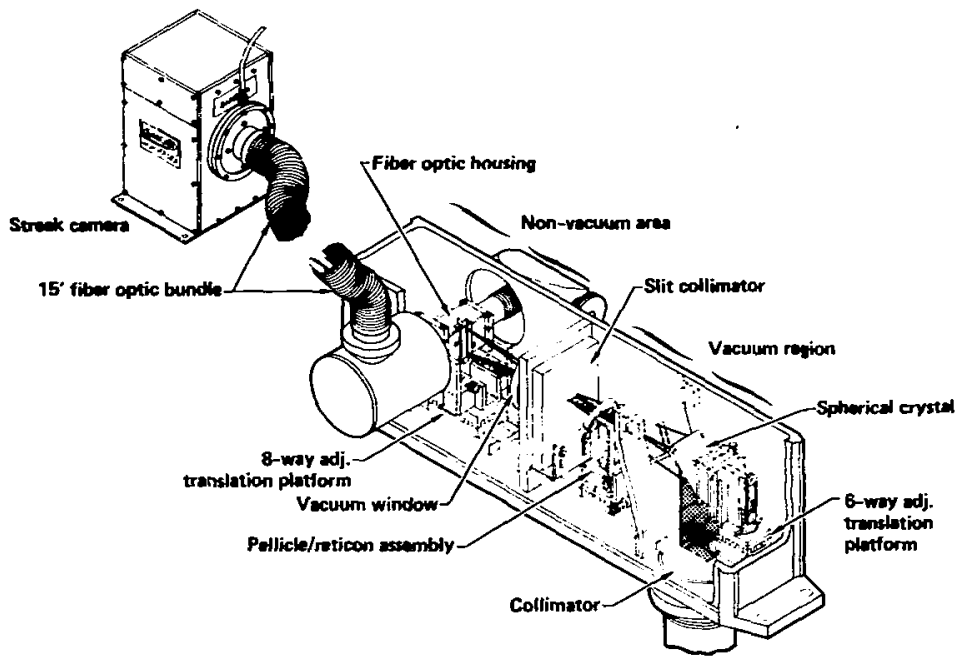

Figure 1. Spherical crystal spectrometer and $\mathbf{x}$-ray detection system.

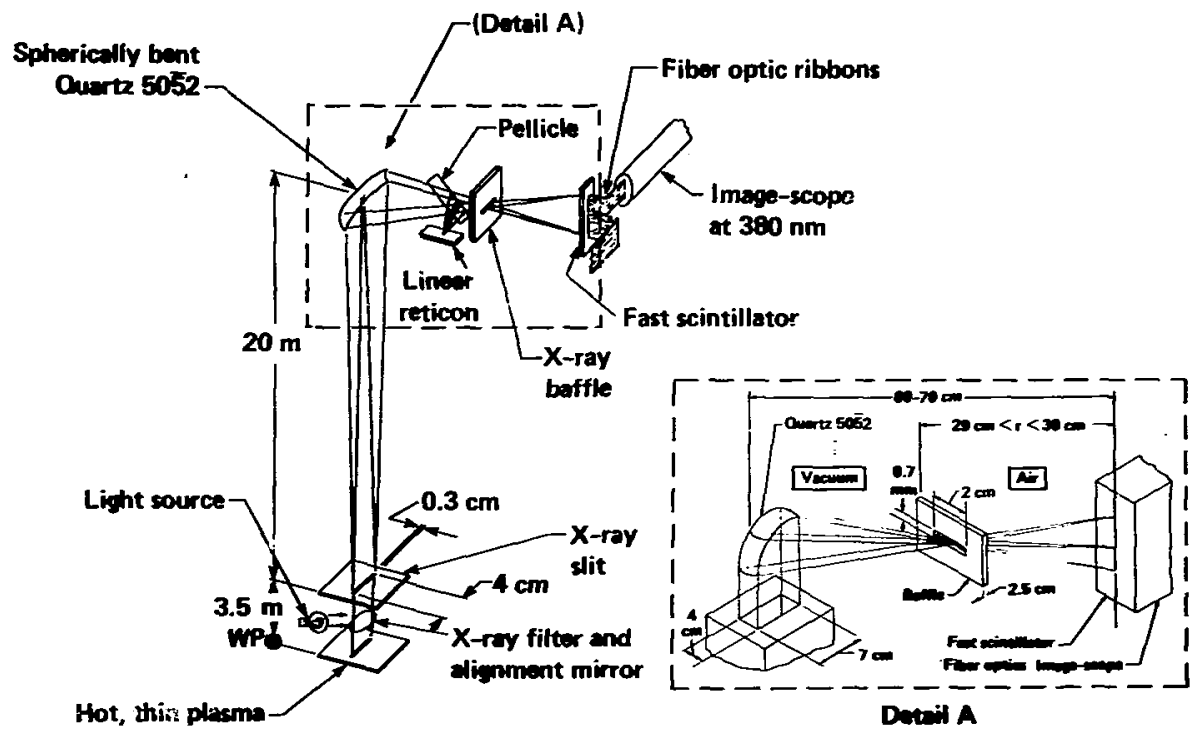

Figure 2 Spherically co:ved crystal spe-trometer experiment, with a sketch of the spherical crystal optica (Detail A). The scintillawor consists of fast phosphor on a radiation-hardened fiber cptic faceplate. The pellicle and linear reticon ane not ahown in the detail. 
$0.3 \times 4.0 \mathrm{~cm}$, and the magnification was roughly $1 / 30$, the spectrum appeared as a line $1.33-\mathrm{mm}$ wide. The !ength of the line was determined by the size of the streak camera photocathode $(3 \mathrm{~cm})$, and this parameter in tum helped to determine the length of the crystal (10 cm).

The angular dispersion can be used with the Bragg equation to find the resolving power of the spectrometer.

$$
\mathrm{d} \lambda / \lambda=\mathrm{dE} / \mathrm{E}=\operatorname{ctn} \theta \mathrm{d} \theta \text {. }
$$

For this particular instrument, $\operatorname{ctn} \theta \simeq 1$. Thus to obtain a resolving power of 10,000 or more the value for $d \theta$ must be less than 1 mrad. Canister deflections on the order of tens of mrad have been measured at the NTS, and since such deflections will influence calibration, 1 needed to do all of the final alignment in the field. For the same reason, it was also necessary to monitor alignment during emplacement.

\section{$X$-ray tube design}

Since the spectrometer had true focusing any calibration using less than a full length $(20 \mathrm{~m})$ line of sight could be used only in testing the procedures that were to be used at the NTS. The actual calibration could tot be done using the $7-\mathrm{m}$ line of sight that was constructed for laboratery work. The $x$-ray source was designed for use on the horizontal line of sight at the laboratory as well as for the vertical line at the NTS.

For field calibration I decided to use a signal intensity of $1600 \times$-rays per second incident on a piece of photographic film at the detector plane. For the brightest lines, such an intensity would give an adequate spectrum in a reasonable amount of time. The minimum source intensity could then be arrived at by dividing this signal intensity by the efficiency of the spectrometer.

I derive the effective angle subtended by a spherical crystal as

$$
d \Omega=I(R \sin \theta / r) w / r
$$

where:

$$
\begin{aligned}
\mathbf{I} & =\text { integrated reflectivity, } \\
\mathbf{w} & =\text { crystal width, } \\
\mathbf{r} & =\text { the crystal-source distance. }^{1}
\end{aligned}
$$

Entering numerical values $\left(I=1.6 \times 10^{-5}, \mathrm{r}=20 \mathrm{~m}, \mathrm{R}=88 \mathrm{~cm}, \mathrm{w}=5 \mathrm{~cm}\right)$ gives a solid angle of $1.2 \times 10^{-9}$.

Multiplying the source intensity by $d \Omega$ gives the diffracted $x$-ray flux. The efficiency curves given by Birks et al. suggest that for an electron-impact energy of $20 \mathrm{keV}$ on (for example) copper, I could expect an efficiency on the order of $10^{-1}-10^{-5}$ photons/sr/electron. ${ }^{3}$ Using $10^{-5}$ for the efficiency, I could expect a throughput of about 1000 photons per $100 \mathrm{~mA}$.

In the design of an $x$-ray calibration source, it must be remembered that the area of the source is determined by the area of the slit (roughly $1\left(\mathrm{~cm}^{2}\right.$ ) and that it is necessary to use lines other than the Ka lines (in some cases, the intensity of these weaker lines was down by two orders of magnitude). Therefore it seemed expedient to design a source capable of focusing at least $100 \mathrm{~mA}$ of 20 -keV electrons onto a target in the shape of a thin line. The line focus, even though it was not altogether necessary for spectrometer calibration, nonetheless improved the source efficiency and eliminated the need for a slit.

The cathode was a 0.25 -man tungsten wire on a one-dimensionai Pierce lens, and the anode was liquid-cooled with pure ethylene-glycol. The dielectric properties of this coolant (which was recirculated through an automobile radiator) were adequate provided that a plastic hose at least 2-m long was used between the radiator (which was grounded) and the anode. We tried other fluorocarbons, but, due either to radiation or themal damage, these compounds would all decompose.

Figure 3 shuws a sketch of the source as it was fielded, and the inset of this figure reproduces a pinhole $x$-ray picture of the line focus. $A$ photograph of the source attached to the calibration line of sight is shown in Figure 4 , with the spectrometer visible in the background. Figure 5 is a photograph of the spectrometer at the NTS.

\section{Calibration procedure}

The calibration consisted of two distinct procedures: laboratory calibration and final calibration in the field.

The laboratory procedures entailed the measurement of resolving power and efficiency. One way of measuring the resolving power was to measure the flat crystal rocking curves in a conventional double crystal spectrometer. A second value was obtained using the spherically bent crystal in the 7-m line of sight to measure the Ge Kal and $\mathrm{K} \bullet 2$ line widths. The double crystal rocking curves gave a full width half maximum of about $8 \times 10^{-5} \mathrm{rad}$, which would imply a resolution of about $800 \mathrm{MeV}$ for the Ge Ka lines. A sample spectrum obtained from Ge with this spectrometer is shown in Figure 6. The FWHM of these lines is essentially the natural width, $3.5 \mathrm{eV}$.

The efficiency was checked by comparing the incident flux with the diffracted flux. This was done using the following procedure: the spectrometer was set up with a sheet of polaroid film to obtain a spectrum of a few known lines. A slit placed directly in front of the film was positioned to let these lines through. Then another spectrum was taken. This time, the spectrum was exposed long enough to see the shadow of the edges of the slit. Then the film holder was replaced with a NaI(Th) detector. A High Purity Ge spectrometer was placed directly in the incident x-ray beam, slightly below the crystal. The source was turned on and a spectrum was obtained in both the Ge and NaI(Th) detectors. The width of the slits in front of the Nal(Th) detector was obtained from the $x$-ray film and was converted into an energy width. Finally, the number of $x$-rays in this energy interval as detected by the Ge spectrometer was compared to that detected by the NaI(Th) detector normialized to their areas. This procedure was performed for numerous lines at various anode voltages, resulting in varied ratios of continuum to line radiation, and giving an integrated reflectivity of $1.6 \times 10^{-5}$ (accurate to about $10 \%$ ). 


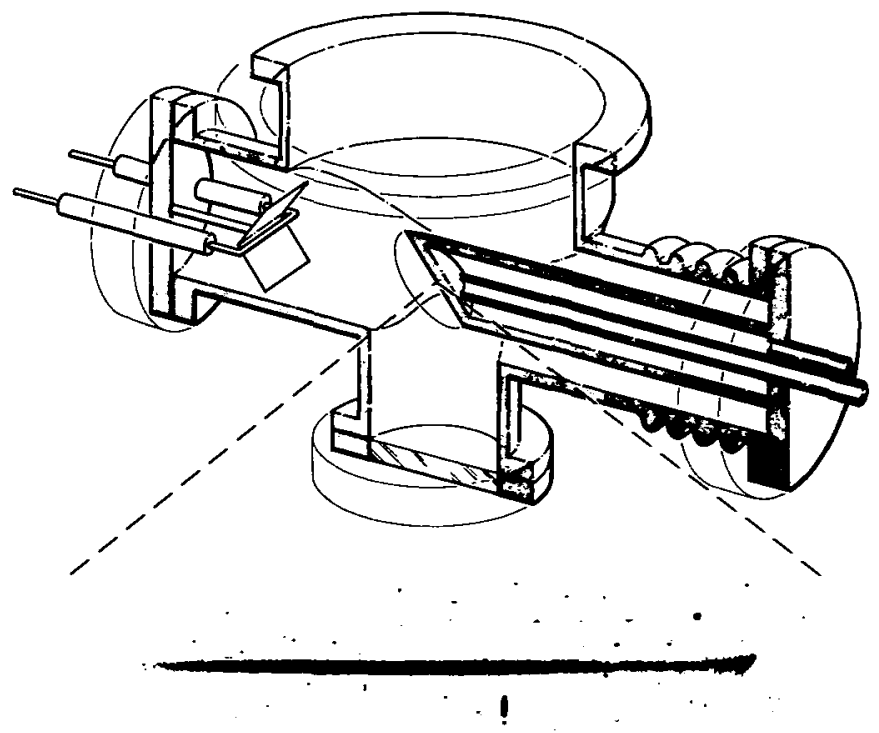

Figure 3. Cutaway view of the $x$-ray source. A pinhole picture of the line focus is given in the inset. The line measures approximately $800 \mu \times 2 \mathrm{~cm}$.

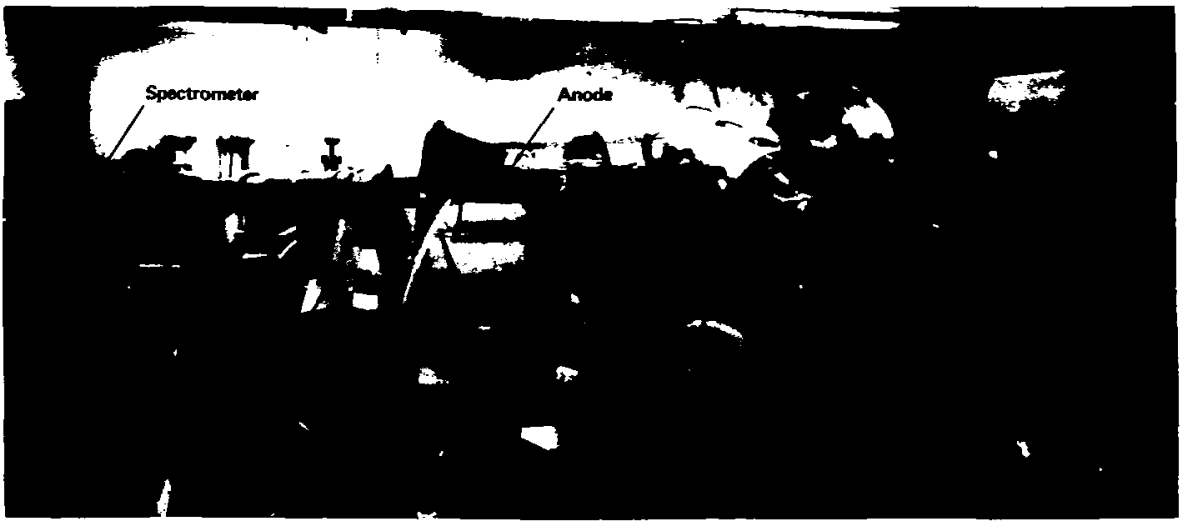

Figure 4. $X$-ray source and calibration line of sight. The end of the anode with its high tension leacls and rubber boved ful of ethylene glycol is visible directly alongride the ion gauge- 


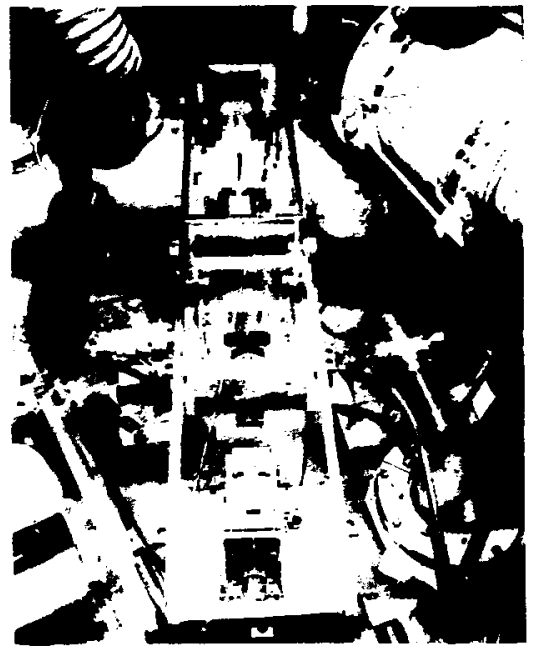

Figure 5. Spectrometer at the NTS.

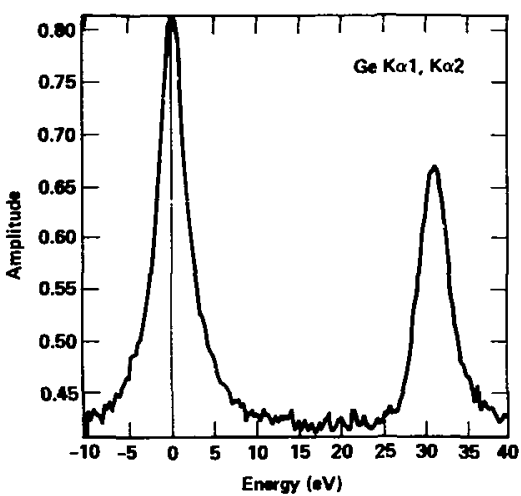

Figure 6. Ge Ka spectrum, measured in the laboratory.

The final dispersion measurements and the final alignment were done at the NiS using the equipment and procedure Jescribed below.

Figure 7a shows a photograph of the $x$-ray detector and film cassette that were used for calibration and alignment. Inside the aluminum box are two fiber optic ribbons. Both are bent $90 \mathrm{deg}$ and $i e d$ visible light from the scintillator to the image-scopos. (These scopes are $\mathbf{l}_{\mathrm{eld}}$ in place at both sides with the translation platform shown in Figure 1.) The sointillator, which is the white strip directly in front of the aluminum box, consists of a fast blue phosphor (Westinghouse w11201) deposited on a fiber optic substrate. The black strip to the right of the aluminum box is the film cassette that was used to do the final alignment and dispersion measurements. This film cassette was made to the exact dimensions of the scintillator. Figure $7 b$ is an example of a calibration spectrum taken at the test site using a platinum-iridium plated anode.
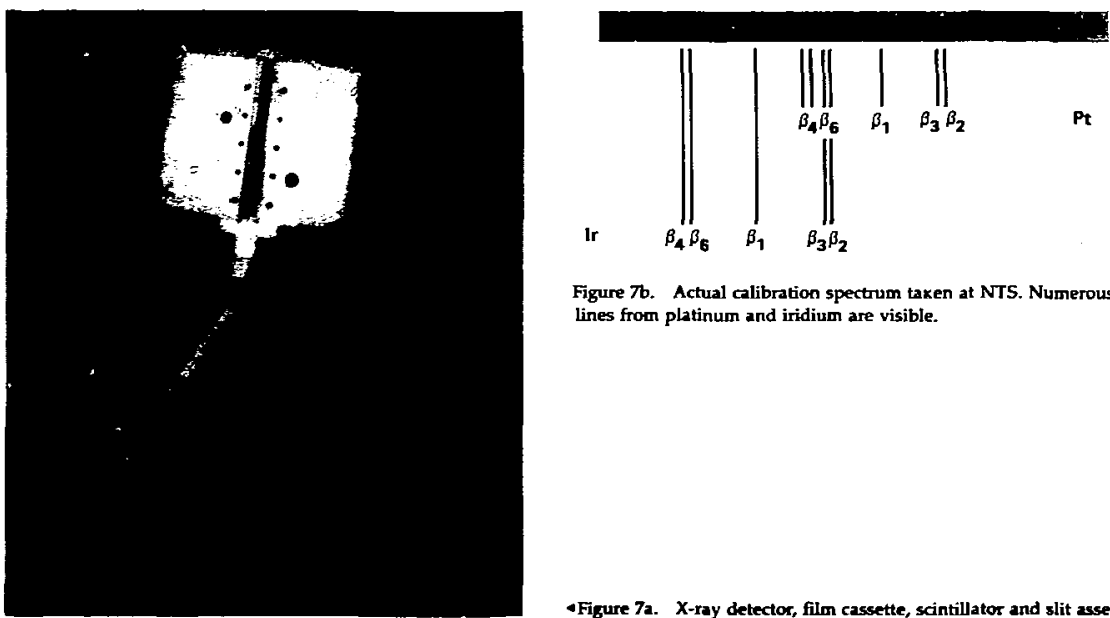

Figure 7b. Actual calibration spectrum taxen at NTS. Numerous $\beta$ lines from platinum and iridium are visible.

-Figure 7a. X-ray detector, film cassette, scintillator and slit assembly. 


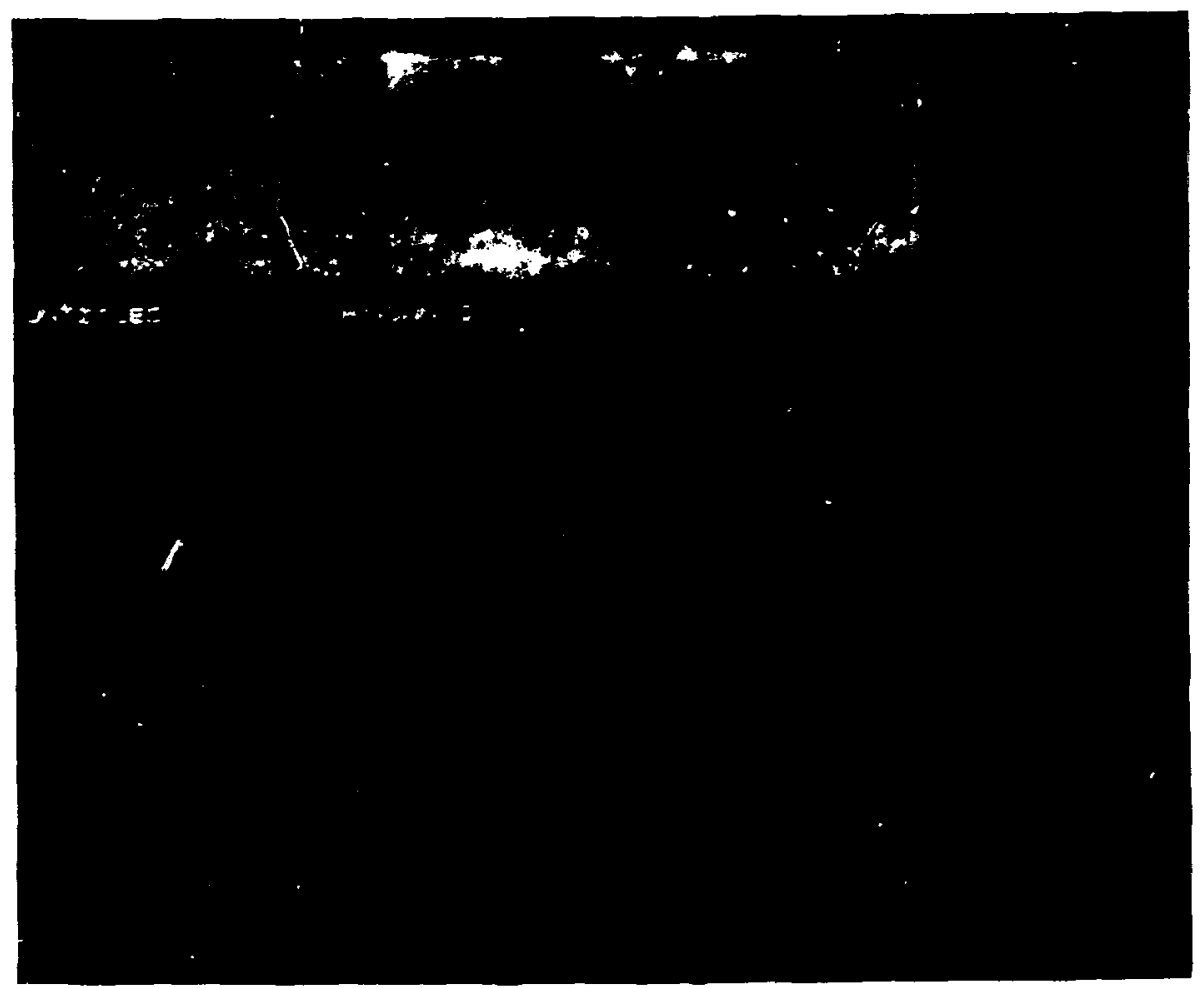

Figure 8. Streak camera data: time nuns vertically from top to bottom, and photon energy increases from left to right. A line at Row 17 is displayed underneath the streak.

Figure $B$ gives the raw data taken from one of the streak cameras. The data consists of about 400 coluans (x-ray energy) and 122 rows (time). The $x$-ray signal is seen running along the top edge of the picture. The red line at row 17 (displayed in the lower half of the figure) provides an example of the richness of the spectra from these types of plasina.

\section{Cenclenien}

1 have demonstrated that very high resolution $x$-ray spectra are possible in a reunote and hostile environment. In order po meke such precise measurements, however, it is necessary to calibrate the instrument in sitw. Moreover, provisions must be nade to check and (if neceesary) make corrections to the calibration at shot time.

The $x$-ray source worked satisfactorily throughout the experiments, but experience hws shown the need of several modifications. For example, the high voltage anode requires a great deal of cooling and the coolant ho to be stable wher it is abjected to ratiotion and hiph tea-

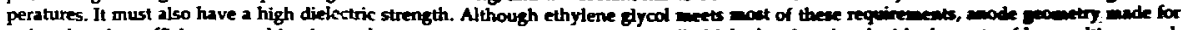

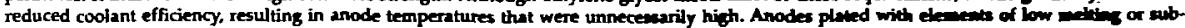
limation temperatures had a very limited lifetime.

Taking these factors into account, I developed a new source design. (A photograph of the as-built tube is shown in Figure 9.) Writh an increase in anode cooling, the new source should be able to accommodate an operating power density of $2 \mathrm{~kW} / \mathrm{cm}^{2}$ at $m$ abode waperature of 


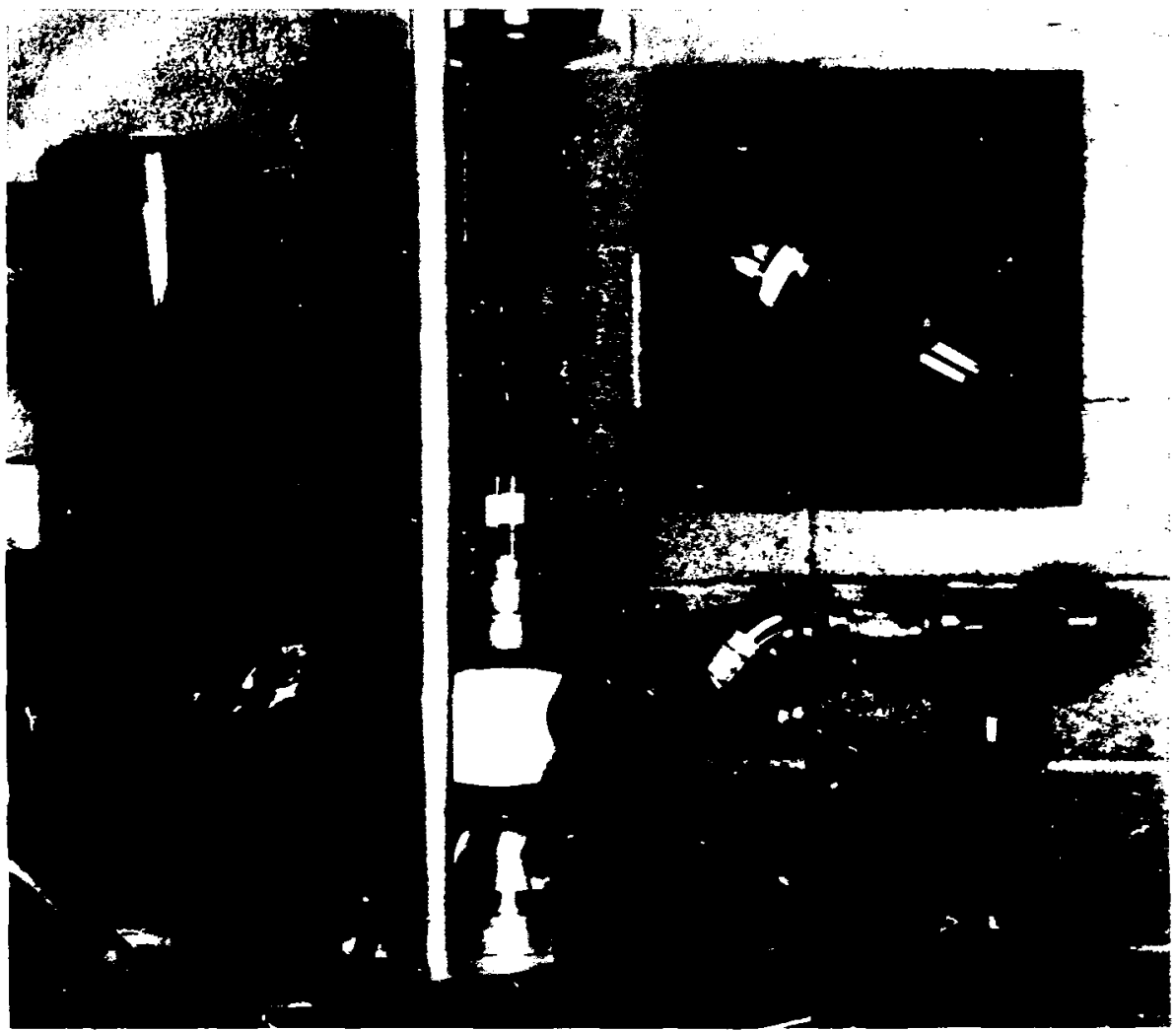

Figure 9. The as-built tube. A picture of the cathode appears in the inset.

$870 \mathrm{~K}$. The anode for this source is grounded, thereby eliminating the need for a dielectric coolant. The cathode (shown in the inset of Figure 9) is in most respects similar to the one sketched in Figure 3. Testing of this tube is just getting under way, but preliminary results are encouraging.

\section{Acknowledgments}

I would like to thank Carl Bruns and Ray Kahle for assembling the hardware and devoting the long hours that were needed to make it work. The work leading to this paper was periormed under the auspices of the U.S. Department of Energy by Lawrence Livermore National Laboratory under contract No. W-7404-Eng-48.

\section{Refereaces}

1. Thoe, R., “High Resolution Spectrometer Design Using Spherically Curved Crystals,” forthcoming in J.O.S.A.

2. Burton, E., Principle and Practice of X-Ray Spectrometric Analysis, Plenum Press, Fp. 201-214. 1970.

3. Birks, L. S., R. E. Seebold, A. P. Batt, and J. S. Grosso, "Excitation of Characteristic X-Rays by Protons, Electrons, and Primary X-Rays," f. Appl. Phys., Vol. 35, No. 9, pp. 2578-2581. 1964. 


\section{DISCTAIMER}

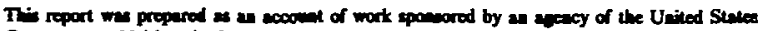

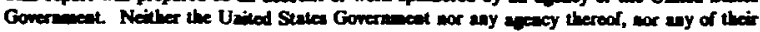

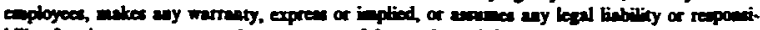

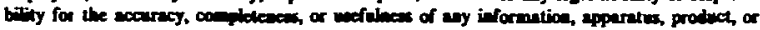

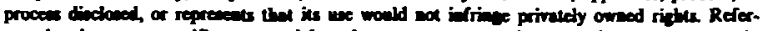

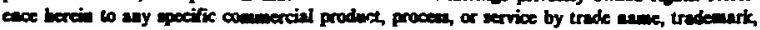

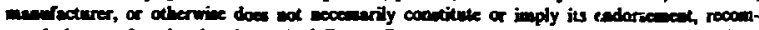

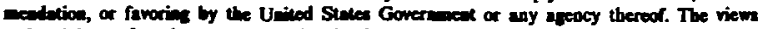

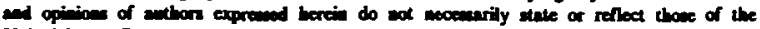
Univad Stave Goveriment or ey apacy thereof. 